\title{
Producción y CARACTERIZACIÓN DE CARbón ACTIVAdo A PARTIR DE RETAMO ESPINOSO (ULEX EUROPAEUS)
}

\author{
Production AND CHARACTERIZATION OF ACTIVATED \\ CARBON OF GORSE (ULEX EUROPAEUS)
}

$\bullet$

\author{
Harvey Andrés Milquez-Sanabria \\ Universidad Nacional Abierta y a Distancia, Escuela de Ciencias Básicas, \\ Tecnología e Ingeniería Bogotá, Colombia
}

Recibido: $14 / 04 / 2017 \cdot$ Aprobado: 25/0572017

\section{RESUMEN}

El retamo espinoso es una de las 100 especies invasoras más dañinas del mundo. En Colombia esta planta fue introducida como cerca viva y se encuentra diseminada por la región Andina. Afecta las cuencas de agua, flora y fauna nativa y es biomasa con un alto poder calorifico, propicia como combustible en incendios forestales. Las acciones de erradicación se concentran en la eliminación manual y mecánica, cuyos residuos son incinerados o puestos en pilas de compostaje. Estas alternativas generan un alto consumo de recursos materiales, financieros y laborales; las cuales son asumidos por entidades distritales, regionales y nacionales, sin embargo, la erradicación total aún está lejos. Por otra parte, el carbón activado tiene una amplia aplicación industrial, y se puede fabricar a partir de diferentes residuos lignocelulósicos. Colombia tiene un consumo anual promedio de 700 tonlaño, con un precio que varía entre 0.5 y 3 USD/kg. La activación de la materia prima carbonosa se puede hacer vía quimica o física; la vía química requiere de la determinación del tipo de ácido o base, concentración, temperatura y tiempo de proceso. El objetivo del presente estudio es producir carbón activado a partir de retamo espinoso, identificando condiciones para obtener un carbón de calidad comercial. Las mejores condiciones encontradas fueron: 1) uso del tallo de la planta, H3PO4 a 50\% v/v, temperatura de activación $550^{\circ} \mathrm{C}$ y tiempo $0.52 \mathrm{~h}$, para producir un carbón activado tipo II macro poroso con indice de yodo de $770.5 \mathrm{mg} / \mathrm{g}$ carbón, área superficial $717.9 \mathrm{~m} 2 / \mathrm{g} y$ densidad aparente $0.47 \mathrm{~g} / \mathrm{ml}$.

Palabras clave: carbón activado, combustible, especie invasora, retamo espinoso, ulex europaeus.

\section{Abstract}

Gorse is one of the 100 species most dangerous in world. In Colombia was introduced from Europe as living fence and nowadays, is scattered by Andes region. It affects rivers, flora, fauna and it is biomass with a high calorific value, suitable as fuel for wildfires. Eradication actions focus on manual and mechanical removal, whose waste is incinerated or put into compost piles. These alternatives generate a high consumption of material, financial and labor resources; which are assumed by district, regional and national entities, however, the total

harvey.milquez@unad.edu.co,orcid.org/0000-0003-4515-713 
eradication is still far away. Activated carbon has a wide industrial application, and can be manufactured from different lignocellulosic residues. Colombia has an average annual consumption of 700 tons/year, with a price that varies between 0.5 and 3 USD/kg. Activating the carbonaceous feedstock can be made by chemical or physical way, chemical route requires the determination of the type of acid or base, concentration, temperature and time. The aim of this study is to produce activated carbon from gorse, identifying conditions to obtain a commercial grade activated carbon. The best conditions found were: 1) use of the plant stem, H3PO4 50\% v/v, activation temperature $550{ }^{\circ} \mathrm{C}$ and $0.52 \mathrm{~h}$ period to produce a type II macroporous activated carbon with an iodine index of $770.5 \mathrm{mg} / \mathrm{g}$ coal, Surface Area $717.9 \mathrm{~m} 2 / \mathrm{g}$ and density $0.47 \mathrm{~g} / \mathrm{ml}$.

Key words: activated carbon, fuel, invasive species, ulex europaeus.

\section{INTRODUCCIÓN}

El retamo espinoso, también conocido como espinillo, argoma o tojo (Ulex europaeus) es una planta nativa europea, incluida en la lista de las 100 especies invasivas más dañinas del mundo. En la actualidad se encuentra en América, Asia, Oceanía, Europa y África. El retamo espinoso es una planta perenne, crece formando áreas densas e impenetrables; es altamente competitivo, desplaza plantaciones artificiales y nativas, es capaz de alterar las condiciones del suelo para ser una especie fijadora de nitrógeno y difícil de erradicar debido a la viabilidad prolongada de la semilla (Lowe et al., 2004).

En Colombia, el retamo espinoso, se encuentra en la región Andina, principalmente en los departamentos de Antioquia, Cundinamarca y Boyacá, afectando las cuencas de agua, fauna y flora nativa y convirtiéndose en combustibles para incendios forestales (Torres Rodríguez, \& Vargas Ríos). La legislación colombiana prohíbe la plantación, trasplante, venta, distribución y comercialización del retamo espinoso, por ser causante de diversos problemas ambientales y sociales, relacionados con el agua, quemas y sequía (CAR, 2009; Minambiente, 2008).

Con la finalidad de erradicar dicha especie, se han planteado diferentes acciones entre las cuales se cuentan: compostaje (Buitrago, 2013), plantación de especies nativas (Lowe et al., 2004), eliminación manual, eliminación mecánica, control químico, control biológico, pastoreo y quemas controladas (Buitrago, 2013b). Los estudios para aprovechar esta especie son escasos, incluyen bioetanol (Ares-Peón, 2013) y biochar (Kaal, 2012).

El carbón activado es un material de estructura porosa, que le confiere elevada capacidad de adsorción (Pediamecum, s.f.; Rodríguez Reinoso, 2017). Sus aplicaciones son: purificación de agua, remoción de compuestos orgánicos y/o tóxicos, eliminación de color, olor y base de catalizadores. La activación del carbón requiere dos elementos primordiales, la fuente de carbono y el método de activación. El método de activación puede ser químico o físico (Zapata Benabithe et al., 2005; Rincón et al., 2015).

La activación química consiste en el aumento de temperatura entre $500-700^{\circ} \mathrm{C}$ en un ambiente sin oxígeno, de la materia prima que fue previamente impregnada con agentes químicos (ácido fosfórico, ácido sulfúrico o cloruro de zinc). Por su parte, la activación física inicia con la carbonización de la materia prima, en donde se eliminan elementos como el hidrógeno y el oxígeno, y continua con la activación en ambiente con vapor de agua, aire o $\mathrm{CO}_{2}$, a temperaturas entre 700 y $1.100{ }^{\circ} \mathrm{C}$ (Bastidas et al., 2010; Rincón-Silva et al., 2014).

La preparación de carbón activado usando materias primas alternativas es un área de diferentes estudios 
basados en residuos agroindustriales: naranja (Реña, Giraldo \& Moreno, 2012), cáscara de palma de aceite (Gómez, Rincón \& Wiest, 2004), cuesco de palma (Gómez, Rincón \& Klose, 2010; Abechi et al., 2013), cáscara de coco (Luna et al., 2007), residuos de manzana (Hesas, et al., 2013, especies forestales (Herrera Builes, Morales Yepes \& Pérez Schile, 2004; RincónSilva et al., 2014) , guadua (Prías Barragán et al., 2011), bambú (Velázquez-Trujillo, Bolaños-Reynoso, \& Pliego-Bravo, 2010), lodos activados (Al-Qodah \& Shawabkah, 2009) y (Monsalvo, Fernandez Mohedano \& Rodriguez, 2011); entre otras fuentes. El objetivo de este estudio es evaluar el uso del retamo espinoso (Ulex europaeus), como materia prima para la elaboración de carbón activado a través de un proceso químico.

\section{Materiales y MÉtodos}

Se recolectó el retamo espinoso en las faldas del cerro de Monserrate, ubicado en la parte oriental de la ciudad de Bogotá, Colombia. La planta se desenterró para liberar las raíces, teniendo mucho cuidado de depositarla en bolsas plásticas sin diseminar semillas. La materia prima recolectada se deja secar al sol por 24 horas. A los tallos se les redujo su tamańo para facilitar el procesamiento mediante una trozadora de disco de carbono de 40 dientes. El material se procesó en un molino de discos y se tamizó hasta tener un precursor homogéneo entre 5 y $10 \mathrm{~mm}$ de diámetro. Luego se impregnó con $\mathrm{H}_{3} \mathrm{PO}_{4}$, se realizaron dos procesos de secado, el primero a $110^{\circ} \mathrm{C}$ por $1 \mathrm{~h}$ y el segundo a $150{ }^{\circ} \mathrm{C}$ por $1.5 \mathrm{~h}$.

La activación se llevó a cabo en un reactor de $3 \mathrm{~L}$ de volumen efectivo, con temperatura máxima de 1.000 ${ }^{\circ} \mathrm{C}$, termocupla tipo $\mathrm{K}$, lana como aislante y recubierto con arena, dos puertos para la entrada y salida de nitrógeno. Para finalizar se lavó el producto con agua destilada hasta asegurar un $\mathrm{pH}$ neutro.

En la primera parte del proyecto se analizó la influencia de la parte del arbusto utilizada (grupo 1 $\left[G_{1}\right]$ : hojas, semillas y frutos, grupo $2\left[G_{2}\right]$ : tallos) y la concentración de ácido fosfórico (50 - 67.5 y 85\% $\mathrm{v} / \mathrm{v}$ ), en la activación química, usando como parámetros de respuesta el índice de yodo, humedad y $\mathrm{pH}$ de la solución resultante. El esquema utilizado se muestra en la Tabla 1.

TABla 1.

Condiciones experimentación fase 1 .

\begin{tabular}{|c|c|c|}
\hline Prueba & Parte arbusto & Concentración $\mathbf{H}_{3} \mathbf{P O}_{4}(\%)$ \\
\hline$P_{1}$ & $G_{1}$ & 50 \\
\hline$P_{2}$ & $G_{1}$ & 67.5 \\
\hline$P_{3}$ & $G_{1}$ & 85 \\
\hline$P_{4}$ & $G_{2}$ & 50 \\
\hline$P_{5}$ & $G_{2}$ & 67.5 \\
\hline$P_{6}$ & $G_{2}$ & 85 \\
\hline
\end{tabular}

En la segunda etapa, se tomó el mejor resultado de la primera y se modificó la temperatura (entre 382 y $718^{\circ} \mathrm{C}$ ), y el tiempo de activación (entre 0.52 y $2.68 \mathrm{~h}$ ), usando un diseño de experimentos central compuesto tipo ortogonal, el diseño se muestra en la Figura 1 en donde la primera coordenada es la temperatura $\left({ }^{\circ} \mathrm{C}\right)$ y la segunda el tiempo (h).

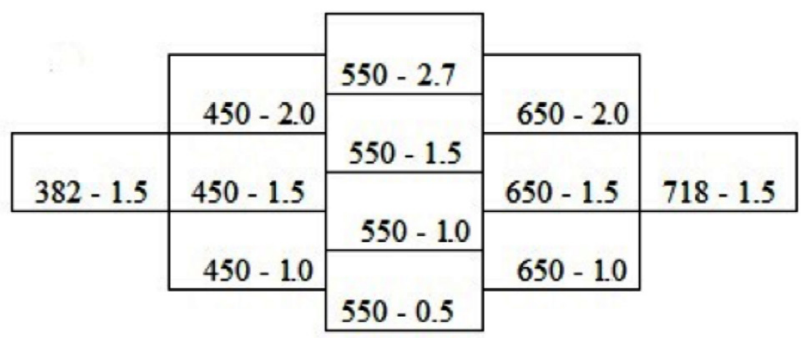

Fig 1. Diseño de superficie central ortogonal

\subsection{Caracterización de la materia prima y del carbón activado}

Las características que se determinaron para el carbón activado obtenido fueron: índice de yodo, humedad y pH; de acuerdo a lo planteado por la Norma Técnica Colombiana NTC 4467, para Carbón Activado de Uso Industrial (Icontec, 1998). El mejor carbón 
activado obtenido en la etapa 2 se analizó mediante: área superficial BET (ASTM D3037), volumen de poro DR, HK y SF (ASTM C699), densidad aparente (ASTM D2854) y la isoterma de adsorción con $\mathrm{N}_{2}$ a $77 \mathrm{~K}$.

\section{Resultados Y DisCUSióN}

\subsection{Etapa 1}

En la Tabla 2 se presentan los resultados obtenidos tras la caracterización del carbón activado preparado en la fase 1 . El experimento $\mathrm{P}_{4}$, que consistió en $50 \%$ de $\mathrm{H}_{3} \mathrm{PO}_{4}$ y el uso de los tallos, presentó las mejores características representadas en mayor índice de yodo (777.6), menor humedad (5.5\%) y pH más cercano a la neutralidad (2.7); las cual confieren propiedades para su uso como agente filtrante en aguas residuales o de proceso, de acuerdo a lo reportado en la literatura en los estudios de: Li et al. (2011), quienes elaboraron carbón activado a partir de residuos lignocelulósicos; por su parte, Diao, Walawender \& Fan (2002), usaron granos de sorgo y activación con ácido fosfórico; y Girgis y El-Hendawy quienes usaron como materia prima semillas de dátiles (2002).

TABla 2.

Caracterización carbón activado etapa 1.

\begin{tabular}{|c|c|c|c|}
\hline Prueba & Índice de yodo $(\mathbf{m g} / \mathbf{g}$ Carbón) & Humedad (\%) & pH \\
\hline$P_{1}$ & $541.7 \pm 1.5$ & $7.6 \pm 0.4$ & $1.8 \pm 0.1$ \\
\hline$P_{2}$ & $551.5 \pm 2.4$ & $7.2 \pm 0.2$ & $1.7 \pm 0.2$ \\
\hline$P_{3}$ & $609.5 \pm 1.8$ & $7.1 \pm 0.4$ & $2.7 \pm 0.2$ \\
\hline$P_{4}$ & $777.6 \pm 3.2$ & $5.5 \pm 0.5$ & $2.2 \pm 0.2$ \\
\hline$P_{5}$ & $741.1 \pm 5.1$ & $8.5 \pm 0.4$ & $2.1 \pm 0.2$ \\
\hline$P_{6}$ & $553.6 \pm 3.3$ & $6.8 \pm 0.2$ & \\
\hline
\end{tabular}

Fuente: autor

\subsection{Etapa 2.}

La Tabla 3 resume los resultados de las pruebas de caracterización del carbón obtenido a las condiciones descritas en la Figura 1, pertenecientes a la etapa 2 del proyecto. Para analizar la variación en el índice de yodo, humedad y $\mathrm{pH}$ debido a la temperatura (A) y tiempo (B), se plantea un análisis de varianza (ANOVA), mediante la herramienta StatGraphics, cuyos resultados se resumen en la Tabla 4.

TABLA 3.

Caracterización carbón activado etapa 2.

\begin{tabular}{|c|c|c|c|c|c|}
\hline Prueba & $\mathbf{T}\left({ }^{\circ} \mathbf{C}\right)$ & $\mathbf{t}(\mathbf{h})$ & $\begin{array}{c}\text { Índice de yodo (mg/g } \\
\text { de carbón) }\end{array}$ & Humedad (\%) & $\mathbf{p H}$ \\
\hline 1 & $450 \pm 0.5$ & 1 & $676 \pm 1.5$ & $5.3 \pm 0.4$ & $2.8 \pm 0.1$ \\
\hline 2 & $650 \pm 0.5$ & 1 & $518.3 \pm 1.3$ & $6.2 \pm 0.3$ & $2.2 \pm 0.2$ \\
\hline 3 & $450 \pm 0.5$ & 2 & $675.9 \pm 2.5$ & $11.2 \pm 0.7$ & $3 \pm 0.2$ \\
\hline 4 & $650 \pm 0.5$ & 2 & $490.8 \pm 2.9$ & $4.4 \pm 0.2$ & $2.2 \pm 0.3$ \\
\hline
\end{tabular}




\begin{tabular}{|c|c|c|c|c|c|}
\hline Prueba & $\mathbf{T}\left({ }^{\circ} \mathbf{C}\right)$ & $\mathbf{t}(\mathbf{h})$ & $\begin{array}{c}\text { Índice de yodo (mg/g } \\
\text { de carbón) }\end{array}$ & Humedad (\%) & $\mathbf{p H}$ \\
\hline 5 & $718 \pm 0.5$ & 1.5 & $277.4 \pm 1.4$ & $4.1 \pm 0.5$ & $1.8 \pm 0.1$ \\
\hline 6 & $382 \pm 0.5$ & 1.5 & $594.6 \pm 2.4$ & $8.2 \pm 0.8$ & $2.6 \pm 0.3$ \\
\hline 7 & $550 \pm 0.5$ & 0.52 & $770.5 \pm 2.3$ & $6.3 \pm 0.3$ & $2.8 \pm 0.2$ \\
\hline 8 & $550 \pm 0.5$ & 2.68 & $318.5 \pm 1.7$ & $4.3 \pm 0.5$ & $1.9 \pm 0.1$ \\
\hline 9 & $550 \pm 0.5$ & 1 & $515 \pm 2.2$ & $5.5 \pm 0.5$ & $2.1 \pm 0.3$ \\
\hline 10 & $450 \pm 0.5$ & 1.5 & $431.5 \pm 2$ & $4.8 \pm 0.4$ & $1.8 \pm 0.3$ \\
\hline 11 & $550 \pm 0.5$ & 1.5 & $450.1 \pm 1.7$ & $4.9 \pm 0.8$ & $1.8 \pm 0.4$ \\
\hline
\end{tabular}

Fuente: autor

TABLA 4.

Resultados del análisis de varianza de la etapa 2.

\begin{tabular}{|c|c|c|}
\hline \multirow{2}{*}{ Fuente } & \multicolumn{2}{|c|}{ Índice de yodo (mg/g Carbón) } \\
\cline { 2 - 3 } & Razón-F & Valor-P \\
\hline A:T & 5.4 & 0.099 \\
\hline B:Tiempo & 10.43 & 0.042 \\
\hline Fuente & Razón-F & Valor-P \\
\hline A:T & 1.02 & 0.515 \\
\hline B:Tiempo & 0.75 & 0.62 \\
\hline Fuente & Razón-F & Valor-P \\
\hline A:T & 6.29 & 0.081 \\
\hline B:Tiempo & 6.13 & 0.084 \\
\hline
\end{tabular}

Fuente: autor

Se observa en la Tabla 4, que el índice de yodo y el $\mathrm{pH}$ se ven afectados por la variación debido a la temperatura (A) y tiempo (B), con un $90 \%$ de nivel de confianza. Mientras que la humedad no se ve afectada por estas dos variables. En la Figura 2 se muestran los efectos principales para el índice de yodo. Existe una tendencia inversamente proporcional entre la temperatura, el tiempo de activación y el índice de yodo. El tiempo de activación más bajo, en general, produce un carbón activado con mayor índice de yodo. Este comportamiento también esta reportado por Li et al. (2011) para los residuos de arroz, obteniendo que el menor tiempo de activación $(0.5$ h) brinda un área superficial mayor para la activación ácida con $\mathrm{H}_{3} \mathrm{PO}_{4}$ al 50\% (Li et al., 2011). 
Por otro lado, la temperatura no debe subir más allá de $550{ }^{\circ} \mathrm{C}$, debido a la disminución del área superficial generada, este resultado está en concordancia con Diao, Walawender \& Fan (2002) quienes trabajaron con los residuos de sorgo mediante activación química con ácido fosfórico, e indican que la temperatura óptima para su proceso fue de $500^{\circ} \mathrm{C}$, la cual generó mayor número de poros sin poner en riesgo la estructura del carbón.

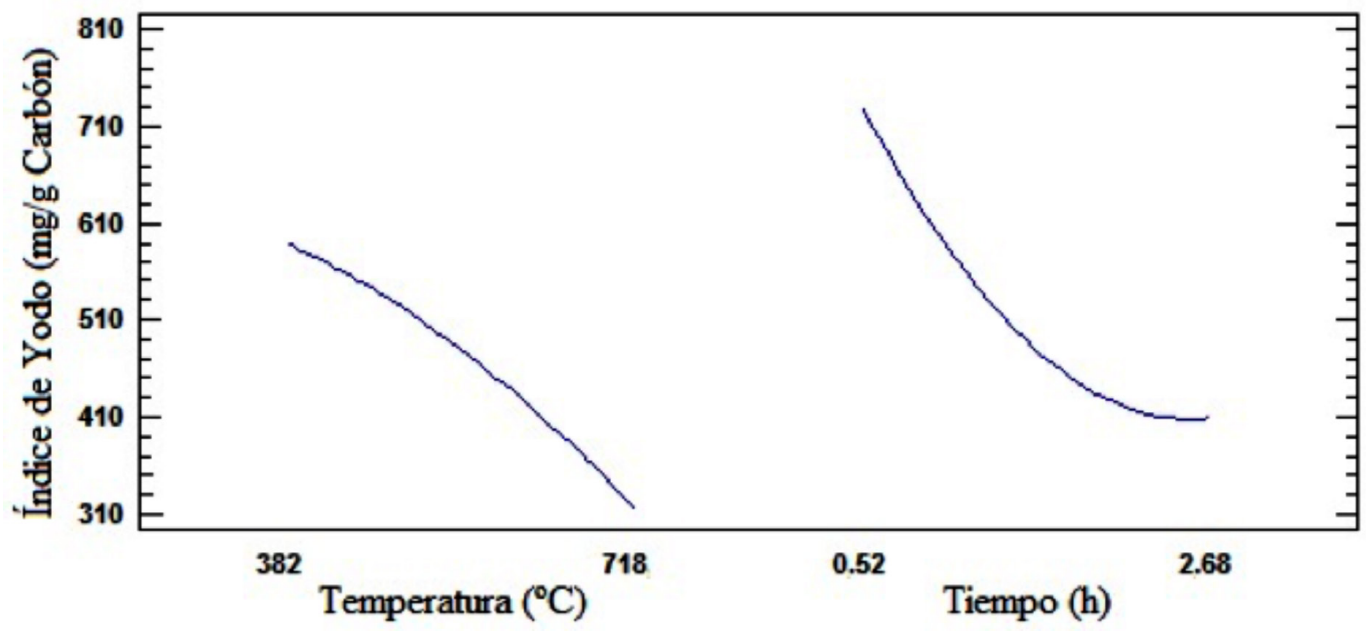

Fig. 2. Efectos principales para el Índice de Yodo

Se planteó un modelo de regresión lineal para describir la relación entre el índice de yodo, la temperatura y el tiempo de activación. El modelo obtenido se presenta en la ecuación 1 , con un coeficiente de ajuste del $55 \%$, lo cual implica que el $55 \%$ en la variación del índice de yodo se explica por la variación en la temperatura y el tiempo de activación.

\section{Ecuación 1.}

Dónde: índice de yodo (mg/g Carbón), T: temperatura $\left({ }^{\circ} \mathrm{C}\right)$, t: tiempo de activación $(\mathrm{h})$.

Se caracterizó el mejor carbón activado obtenido en la etapa 2, el cual consiste en la activación a 550 ${ }^{\circ} \mathrm{C}$ por un $0.52 \mathrm{~h}$, los resultados se pueden apreciar en la tabla 5 , junto con resultados de otros estudios para la producción de carbón activado a partir de residuos agroindustriales. El carbón activado, a partir de retamo espinoso por activación química, presenta densidad aparente menor a la reportada para palmiste por Abechi et al. (2013), pero mayores a la reportada por Velázquez-Trujillo, Bolaños-Reynoso \& Pliego-Bravo
(2010) para bambú. Este factor es de vital importancia en el diseño de columnas de absorción, debido a que es una medida indirecta de la cantidad de ab- sorbato que el carbón puede retener por unidad de volumen (Abechi et al., 2013).

El área superficial del carbón activado a partir de retamo espinoso fue de $717.9 \mathrm{~m}^{2} / \mathrm{g}$, superior a la obtenida para los residuos lignocelulósicos de palmistre por activación química, de acuerdo a lo reportado por Abechi et al. (2013). El área superficial reportada en el actual estudio está en el mismo orden de magnitud que la reportada por otros autores, para los residuos de manzana (Hesas et al., 2013), bambú (VelázquezTrujillo, Bolaños- Reynoso \& Pliego-Bravo, 2010) y cáscara de naranja (Peña, Giraldo \& Moreno, 2012). De acuerdo con estos últimos autores, la adsorción de compuestos en el carbón activado se ve favorecida por dos principales rutas: termodinámicamente por las características ácidas de los grupos funcionales, y cinéticamente por el área específica del adsorbente (Peña, Giraldo \& Moreno, 2012). 


\section{Tabla 5.}

Densidad aparente, área superficial y caracterización de poro para el carbón activado a partir de retamo espinoso y otras fuentes

\begin{tabular}{|c|c|c|c|c|c|}
\hline & $\begin{array}{c}\text { Densidad } \\
\text { aparente }(\mathrm{g} / \mathrm{ml})\end{array}$ & $\begin{array}{l}\text { Área superficial, } \\
\text { BET }\left(\mathrm{m}^{2} / \mathrm{g}\right)\end{array}$ & $\begin{array}{l}\text { Volumen de } \\
\text { poro, DR } \\
\left(\mathrm{cm}^{3} / \mathrm{g}\right)\end{array}$ & $\begin{array}{c}\text { Volumen de } \\
\text { poro, HK } \\
\left(\mathrm{cm}^{3} / \mathrm{g}\right)\end{array}$ & $\begin{array}{c}\text { Volumen de } \\
\text { poro, SF }\left(\mathrm{cm}^{3} / \mathrm{g}\right)\end{array}$ \\
\hline Retamo espinoso & 0.46 & 717.9 & 0.44 & 0.28 & 0.29 \\
\hline Manzana (Hesas et al., 2013) & NR & 854 & 0.45 & 0.32 & 0.13 \\
\hline Palmistre (Abechi et al., 2013) & 0.62 & 217 & 0.12 & 0.11 & 0.01 \\
\hline $\begin{array}{c}\text { Cascarilla de arroz (Carrillo Qui- } \\
\text { jano, Albarracín Caballero } \\
\text { \& Pereira Hernández, 2013) }\end{array}$ & NR & 1713 & 1.789 & 0.72 & 1.07 \\
\hline $\begin{array}{c}\text { Bambú (Velázquez-Trujillo } \\
\text { et al., 2010) }\end{array}$ & 0.23 & 917 & NR & NR & NR \\
\hline $\begin{array}{l}\text { Cascara de naranja (Peña, } \\
\text { Giraldo \& Moreno, 2012) }\end{array}$ & NR & 943 & 0.44 & 0.2 & 0.24 \\
\hline
\end{tabular}

NR: No reportado

Finalmente, en la Figura 3, se presenta la isoterma de adsorción de nitrógeno a $77 \mathrm{~K}$. Según la clasificación de la IUPAC, se obtuvo una isoterma tipo II, característica de sólidos no porosos, dicho comportamiento se debe a temperaturas de activación medias y altas (Balbuena \& Gubbins, 1993). Al ser un carbón activado tipo II macroporoso tiene una alta interacción con aguas contaminadas con arcillas, pigmentos y cementos (Do, 1998).

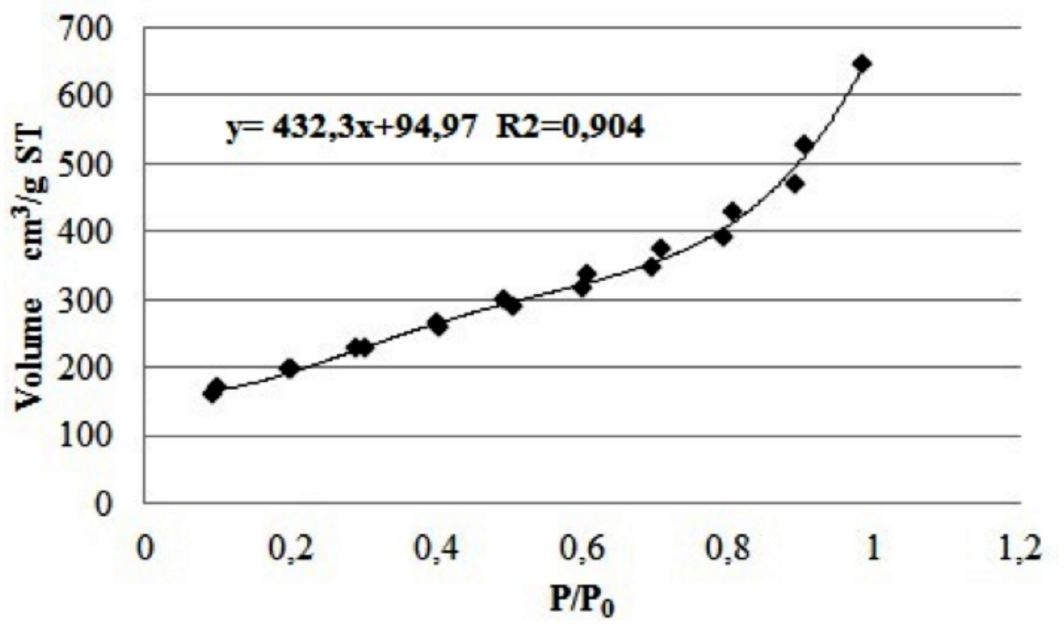

Fig. 3. Isoterma de adsorción $\mathrm{N} 277 \mathrm{~K}$ para carbón activado a $550{ }^{\circ} \mathrm{C}$ y 0.52 h.

\section{CONCLUSIONES Y RECOMENDACIONES}

Se preparó carbón activado a partir de retamo espinoso (Ulex europaeus), utilizando las ramas, semillas y hojas, mediante la activación química con $\mathrm{H}_{3} \mathrm{PO}_{4}$ a tres diferentes concentraciones $(50-67.5$ y $80 \% \mathrm{v} / \mathrm{v})$, a diferentes temperaturas $\left(382,450,550,650\right.$ y $\left.718^{\circ} \mathrm{C}\right)$ y tiempos de activación $(0.52-1-1.5$ y 2.68 h). Se determinaron propiedades del carbón activado incluyendo humedad, $\mathrm{pH}$, índice de yodo, área superficial 
BET, volumen de poro, densidad aparente y la curva de adsorción de $\mathrm{N}_{2}$ a $77 \mathrm{~K}$. Las condiciones óptimas encontradas para obtener la mayor área superficial activa fueron: uso del tallo, $50 \%$ de $\mathrm{H}_{3} \mathrm{PO}_{4}, 550{ }^{\circ} \mathrm{C}$ y $0.52 \mathrm{~h}$.

Es recomendable seguir investigando diversas alternativas para el aprovechamiento del retamo espinoso, y de esta manera ayudar a la erradicación de esta planta invasora de las fuentes hídricas de la Sabana de Bogotá.

\section{REFERENCIAS}

Abechi, S. E. Gimba, C. E. Uzairu, A. \& Dallatu, Y. A. (2013). Preparation and characterization of activated carbon from palm kernel shell by chemical activation. Research Journal of Chemical Sciences, 3(7), 54-61.

Al-Qodah Z. \& Shawabkah, R. (2009). Production and characterization of granular activated carbon from activated sludge. Brazilian Journal of Chemical Engineering, 26(1), 127-136.

Ares-Peón, I. A. Romaní, A. Garrote, G. \& Parajó J. C. (2013). Invasive biomass valorization: Environmentally friendly processes for obtaining second generation bioethanol and saccharides from Ulex europaus. Journal of Chemichal Technology and Biotechnology, 88(6), 999-1006.

Balbuena P. B. \& Gubbins, K. E. (1993). Theoretical interpretation of adsorption behavior of simple fluids in slit pores. Langmuir, 9(7), 1801-1814.

Bastidas, M. Buelvas, L. M. Márquez M. I., \& Rodríguez, K. (2010). Producción de carbón activado a partir de precursores carbonosos del departamento del Cesar, Colombia. Información Tecnológica, 21(3), 87-96.

Buitrago, L. (2013). Protocolo de Bioseguridad para el Manejo del Compost o Sustratos resultante de la transformación de los residuos de retamo espinoso (Ulex europaeus) y retamo liso (Genista monspessulana), para su incorporación a los procesos de propagación de material vegetal Bajo condiciones controladas. Bogotá: Jardín Botánico José Celestino Mutis.

Buitrago, L. (2013b). Producto 5. Propuesta para el manejo, transformación y Aprovechamiento integral y biosegura de los residuos Vegetales del retamo espinoso (Ulex europaeus) y retamo liso (Genista monspessulana) para la región capital. Bogotá: Jardín Botánico José Celestino Mutis.

CAR (2009). Resolución 469 de 2009. Por medio de la cual se prohíbe la plantación, transplante, venta, distribución y comercialización de las especies Retamo Espinoso (Ulex europaeus) y Retamo Liso (Teline monspessulana) y se adoptan otras disposiciones. Recuperado en: www.nuevalegislacion.com/files/susc/ cdj/conc/r_car_469_09.doc
Carrillo Quijano, C. C., Albarracín Caballero, J. \& Pereira Hernández, X. I. (2013). Producción de carbón activado y sílice a partir de cascarilla de arroz - una revisión. Scientia et technica, $18(2), 422-429$

Diao, Y. Walawender, W. P. \& Fan, L. T. (2002). Activated carbons prepared from phosphoric acid activation of grain sorghum. Bioresource Technology, 81(1), 45-52.

Do, D. D. (1998). Adsorption Analysis: Equilibria And Kinetics. Singapore: Imperial Collegue Press.

Girgis B. S. \& El-Hendawy, A.-N. A. (2002). Porosity development in activated carbons obtained from date pits under chemical activation with phosphoric acid. Microporous and Mesoporous Materials, 52(2), 105-117.

Gómez, A. Klose, W. Rincón, S. L. \& Wiest, W. (2004). Proceso de producción de carbón activado a partir de cáscaras de palma de aceite en un horno rotatorio y su aplicación en la limpieza de NO. Palmas, 25, 461-471.

Gómez, A. Rincón, S. \& Klose, W. (2010). Carbón activado de cuesco de palma: estudio de termogravimetría y estructura. Kassel: Kassel University Press GmbH.

Herrera Builes, J. F. Morales Yepes, W. A. \& Pérez Schile, J. D. (2004). Selección de un método para producir carbón activado utilizando cuatro especies forestales. Revista Facultad Nacional de Agronomía Medellín, 57(2), 2501 -2516.

Hesas, R. H. Arami-Niya, A. Daud, W. M. A. W. \& Sahu, J. N. (2013). Preparation and characterization of activated carbon from apple waste by microwave-assisted phosphoric acid activation: application in methylene blue adsorption. BioResources, 8(2), 2950-2966.

Icontec (1998). Productos industriales. Carbón activado. Métodos de ensayo. NTC4467-1998. Recuperado en: https://tienda. icontec.org/wp-content/uploads/pdfs/NTC4467.pdf

Kaal, J. Martínez, A. Reyes O., \& Soliño, M. (2012). Molecular characterization of Ulex europaeus biochar obtained from laboratory heat treatment experiments-A pyrolysis-GC/MS study. Journal of Analytical and Applied Pyrolysis, 95, 205-212.

Li Y., Ding, X., Guo, Y., Wang, L., Rong, C., Qu, Y., Ma, X. \& Wang, Z. (2011). A simple and highly effective process for the preparation of activated carbons with high surface área. Materials Chemistry and Physics, 127(3), 495-500.

Lowe S., Browne M., Boudjelas S., De Poorter M. (2004). 100 de las especies exóticas invasoras más dañinas del mundo. Una selección del Global Invasive Species Database. Publicado por el Grupo Especialista de Especies Invasoras (GEEI), un grupo especialista de la Comisión de Supervivencia de Especies (CSE) de la Unión Mundial para la Naturaleza (UICN). Recuperado en: http://www.iucngisd.org/gisd/pdf/100Spanish.pdf

Luna, D. González, A. Gordon M., \& Martín, N. (2007). Obtención de carbón activado a partir de la cáscara de coco. ContactoS, 64(10), 39-48. 
Ministerio de Ambiente (2008). Resolución 848 de 2008. Por la cual se declaran unas especies exóticas como invasoras y se señalan las especies introducidas irregularmente al país que pueden ser objeto de cría en ciclo cerrado y se adoptan otras determinaciones. República de Colombia. Recuperado en: www. parquesnacionales.gov.co/portal/wp-content/uploads/2013/08/ res_0848.pdf

Monsalvo, V. M. Fernandez Mohedano A., \& Rodriguez, J. J. (2011). Activated carbons from sewage sludge: application to aqueous-phase adsorption of 4-chlorophenol. Desalination, 277(1-3), 377-382.

Pediamecum (s.f.). Carbón activado. Recuperado en: http://pediamecum.es/wp-content/farmacos/Carbon_activado.pdf

Peña, K. J. Giraldo, L. \& Moreno, J. C. (2012). Preparación de carbón activado a partir de cáscara de naranja por activación química. Caracterización física y química. Revista Colombiana de Química, 41(2), 311-323.

Prías Barragán, J. J. Rojas González, C. A. Echeverry Montoya, N. A. Fonthal, G. \& Ariza Calderón, H. (2011). Identificación de las variables óptimas para la obtención de carbón activado a partir del precursor Guadua angustifolia Kunth. Revista de la Academia Colombiana de Ciencias Exactas, Físicas y Naturales, 35(135), 157-166.

Rincón, J. Rincón, S. Guevara, P. Ballén, D. Morales, J. C. \& Monroy, N. (2015). Producción de carbón activado mediante méto- dos físicos a partir de carbón de El Cerrejón y su aplicación en el tratamiento de aguas residuales provenientes de tintorerías. Revista Academia Colombiana de Ciencias Exactas, Físicas y $\mathrm{Na}$ turales, 39(151), 171-175.

Rincón-Silva, N. G. Ramirez-Gomez, W. M. Mojica-Sánchez, L.C. Blanco-Martínez, D. A. Giraldo, L. \& Moreno-Piraján, J. C. (2014). Obtención de carbones activados a partir de semillas de eucalipto, por activación química con $\mathrm{H} 3 \mathrm{PO} 4$. Caracterización y evaluación de la capacidad de absorción de fenol desde solución acuosa. Ingeniería y Competitividad, 16(1), 207-219.

Rodríguez Reinoso, F. (2017). The versatility of carbon materials. Boletín del Grupo Español del Carbón, 45, 2-7

Torres Rodríguez, N. \& Vargas Ríos, O. (2011). Banco de semillas germinable en áreas invadidas por retamo espinoso (Ulex europaeus) con diferentes edades de quema. En: Vargas, o. \& Reyes, S. (eds.). La restauración ecológica en la práctica. (pp.491 - 506). Bogotá: Universidad Nacional de Colombia.

Velázquez-Trujillo, A. Bolaños-Reynoso, E. \& Pliego-Bravo, Y. S. (2010). Optimización de la producción de carbón activado a partir de bambú. Revista Mexicana de Ingeniería Química, 9(3), 359-366.

Zapata Benabithe, Z. Rojas Rodríguez, D. A. Arenas Castiblanco, E. Chejne Janna, F. Londońo Giraldo, C. \& Pérez Schile, J. D. (2005). Producción de carbón activado a partir de carbón subbituminoso en reactores de lecho fluidizado por proceso autotérmico. Dyna, 72(147), 46-56. 
